\title{
Differentiation between Clear Cell Sarcoma of the Kidney and Wilms' Tumor with CT
}

\author{
Choeum Kang ${ }^{1}$, Hyun Joo Shin ${ }^{1}$, Haesung Yoon ${ }^{1}$, Jung Woo Han ${ }^{2}$, Chuhl Joo Lyu ${ }^{2}$, Mi-Jung Lee \\ ${ }^{1}$ Department of Radiology and Research Institute of Radiological Science, Severance Children's Hospital, Yonsei University College of Medicine, \\ Seoul, Korea; ${ }^{2}$ Division of Pediatric Hematology and Oncology, Department of Pediatrics, Severance Hospital, Yonsei University College of \\ Medicine, Seoul, Korea
}

Objective: Clear cell sarcoma of the kidney (CCSK) is the second-most common but extremely rare primary renal malignancy in children after Wilms' tumor. The aims of this study were to evaluate the imaging features that could distinguish between CCSK and Wilms' tumor and to assess the features with diagnostic value for identifying CCSK.

Materials and Methods: We reviewed the initial contrast-enhanced abdominal-pelvic CT scans of children with CCSK and Wilms' tumor between 2010 to 2019. Fifty-eight children (32 males and 26 females; age, 0.3-10 years), 7 with CCSK, and 51 with Wilms' tumor, were included. The maximum tumor diameter, presence of engorged perinephric vessels, maximum density of the tumor (Tmax) of the enhancing solid portion, paraspinal muscle, contralateral renal vein density, and density ratios (Tmax/muscle and Tmax/vein) were analyzed on the renal parenchymal phase of contrast-enhanced CT. Fisher's exact tests and Mann-Whitney $\mathrm{U}$ tests were conducted to analyze the categorical and continuous variables, respectively. Logistic regression and receiver operating characteristic curve analyses were also performed.

Results: The age, sex, and tumor diameter did not differ between the two groups. Engorged perinephric vessels were more common in patients in the CCSK group (71\% [5/7] vs. 16\% [8/51], $p=0.005)$. Tmax (median, 148.0 vs. 111.0 Hounsfield unit, $p=0.004$ ), Tmax/muscle (median, 2.64 vs. 1.67, $p=0.002$ ), and Tmax/vein (median, 0.94 vs. $0.59, p=0.002$ ) were higher in the CCSK compared to the Wilms' group. Multiple logistic regression revealed that engorged vessels (odds ratio 13.615; 95\% confidence interval [CI], 1.770-104.730) and Tmax/muscle (odds ratio 5.881; 95\% CI, 1.337-25.871) were significant predictors of CCSK. The cutoff values of Tmax/muscle ( $86 \%$ sensitivity, $77 \%$ specificity) and Tmax/vein (71\% sensitivity, $86 \%$ specificity) for the diagnosis of CCSK were 1.97 and 0.76 , respectively.

Conclusion: Perinephric vessel engorgement and greater tumor enhancement (Tmax/muscle $>1.97$ or Tmax/vein $>0.76$ ) are helpful for differentiating between CCSK and Wilms' tumor in children aged below 10 years.

Keywords: Pediatric renal tumor; Clear cell sarcoma of the kidney; Wilms' tumor; Image enhancement; Peritumoral vessel

\section{INTRODUCTION}

Clear cell sarcoma of the kidney (CCSK) is a rare pediatric primary renal tumor, but the second-most common primary renal malignancy in children after Wilms' tumor. The annual

Received: March 17, 2020 Revised: October 9, 2020

Accepted: October 13, 2020

Corresponding author: Mi-Jung Lee, MD, PhD, Department of Radiology and Research Institute of Radiological Science, Severance Children's Hospital, Yonsei University College of Medicine, 50-1 Yonsei-ro, Seodaemun-gu, Seoul 03722, Korea.

- E-mail:mjl1213@yuhs.ac

This is an Open Access article distributed under the terms of the Creative Commons Attribution Non-Commercial License (https://creativecommons.org/licenses/by-nc/4.0) which permits unrestricted non-commercial use, distribution, and reproduction in any medium, provided the original work is properly cited. incidence is 20 in the United States [1,2]. The male to female ratio of for the prevalence of CCSK was 2:1 and the mean age at diagnosis was 36 months $[1,3,4]$. The common clinical manifestations of CCSK include a palpable abdominal mass, abdominal pain, hematuria, and bone pain, similar to Wilms' tumor [5,6]. However, the prognosis of CCSK is worse than that of Wilms' tumor, although the use of high-intensity chemotherapy has dramatically improved the outcomes of CCSK $[1,2,6,7]$. Therefore, differentiating between CCSK and Wilms' tumor is essential for treatment planning and predicting the prognosis.

The final diagnosis of CCSK requires histopathological confirmation. CCSK commonly appears as a heterogeneously enhancing mass without specific features on abdominal CT $[5,8]$. These tumors usually contain cystic, hemorrhagic, 
or necrotic components. Calcifications within the tumor are observed infrequently. However, these imaging features of CCSK are non-specific and are similar to other primary renal tumors, especially Wilms' tumors. To date, bone metastasis, which is observed only in advanced cases, is the only known finding that favors a diagnosis of CCSK over Wilms' tumors $[5,9]$. Therefore, the differential diagnosis of CCSK is difficult with respect to other pediatric renal tumors. However, CCSK exhibits a distinctive vascular network histopathologically, which is not prominent in other pediatric renal tumors [10]. No study has analyzed the imaging findings that represent these pathological findings. Therefore, the aims of this study were to evaluate the imaging features that could distinguish between CCSK and Wilms' tumor and to assess the features with diagnostic value for identifying CCSK.

\section{MATERIALS AND METHODS}

\section{Study Population}

The Institutional Review Board of our hospital approved this retrospective study, and the need for informed consent was waived (IRB No. 1-2019-0090). The study population was retrospectively enrolled by conducting a medical chart review of children with renal tumors diagnosed before the age of 10 years. This study included 58 children with renal tumors for the comparison of the imaging features of CCSK and Wilms' tumors: the CCSK group consisted of 7 patients (male:female $=2: 5$ ) and the Wilms' tumor group consisted of 51 patients (male:female $=30: 21$ ). We reviewed the initial imaging studies in patients with Wilms' tumor or CCSK and checked whether contrast-enhanced abdominalpelvic CT was performed or recorded at our hospital between January 2010 and December 2019. The demographic data assessed included sex, age at the time of the CT scan, and pathological findings including lymph node metastasis, presence of distant metastasis, and disease stage. Each patient's follow-up duration and disease status (stable or progressed) at the last follow-up were also recorded.

\section{CT Scan and Image Review}

We included all the registered CT examinations performed at and outside our hospital. Imaging was performed using various $\mathrm{CT}$ machines with different protocols due to the long study duration. Usually, axial and coronal images in the renal parenchymal enhancement phase, with a 3-mm thickness and contrast enhancement at 50-70 seconds delay after contrast injection were selected for the image review. We excluded CT scans without a renal parenchymal enhancement phase or images obtained after any treatment. Therefore, 7 patients from the Wilms' tumor group and 1 patient from the CCSK group were excluded due to lack of pre-treatment CT studies.

One pediatric radiologist with 15 years' experience in this field performed a blind review of the initial CT images. Imaging findings such as maximal tumor diameter on axial or coronal imaging, presence of engorged perinephric vessels or tumor calcifications, internal heterogeneity of the tumor, venous tumor thrombus, and tumor rupture were evaluated (Fig. 1). Engorged perinephric vessels were defined as tortuous vascular dilatation around the kidneys with tumors. The tumor stage was determined on the basis of the presence of lymph node (short diameter $>1 \mathrm{~cm}$ ) or liver metastasis on CT.

The mean tumor density (Tmean) and maximal tumor density (Tmax) of the most enhanced solid portion were measured. The mean density of the paraspinal muscles at the same level as that used for tumor density measurement and mean density of the renal vein of the contralateral kidney were also obtained. A single, circular region of interest was drawn on the most enhanced solid portion of each tumor on the axial imaging to obtain the tumor density values, and the necrotic or hemorrhagic portions, internal vessels, or calcifications were avoided (Fig. 1). Other circular regions of interest were drawn at the paraspinal muscles and contralateral renal veins, and the adjacent soft tissues and beam-hardening artifacts from the vertebrae were avoided. The same pediatric radiologist repeated the measurement to evaluate its reliability, which was performed separately after an interval of more than 1 month.

We calculated the density ratios by dividing Tmean by muscle density (Tmean/muscle) and dividing Tmax by muscle density (Tmax/muscle) to correct for the differences in the enhancement timing. We also calculated the density ratios by dividing Tmean by the contralateral renal vein density (Tmean/vein) and by dividing Tmax by the contralateral renal vein density (Tmax/vein) to eliminate the effect of several factors influencing tumor enhancement.

\section{Statistical Analysis}

Statistical analyses were conducted using SPSS software version 23 (IBM Corp.) and MedCalc Statistical Software 

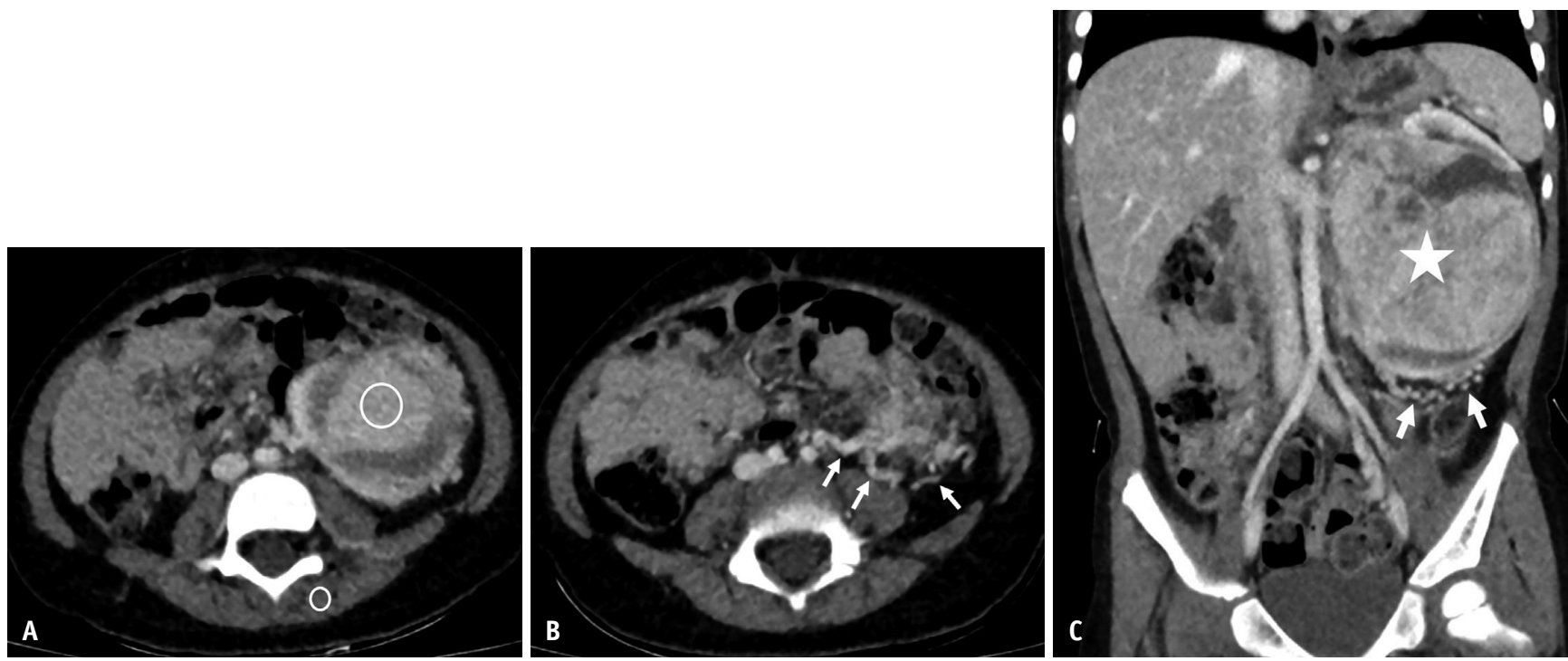

Fig. 1. A 3-year-old girl with a left renal mass confirmed as clear cell sarcoma of the kidney.

A-C. The axial (A, B) and coronal (C) contrast-enhanced abdominal-pelvic CT images in the renal parenchymal phase demonstrate a large, heterogeneously enhancing mass (star) replacing the left kidney tissue with engorged perinephric vessels (arrows) under the left renal tumor. Regions of interest (circles) marked on an axial (A) image were used to obtain the mean (Tmean) and maximum (Tmax) tumor densities of the most enhancing solid portion and mean density of the paraspinal muscle. The Tmean was $212.7 \mathrm{HU}$, Tmax was $277.0 \mathrm{HU}$, paraspinal muscle density was $80.0 \mathrm{HU}$, and contralateral renal vein density was 264.7 HU. Both Tmax/muscle $(3.46>1.97)$ and Tmax/vein $(1.05>0.76)$ were higher than the suggested cutoff values. $\mathrm{HU}=$ Hounsfield unit, Tmax = maximum density of the tumor, Tmax/muscle $=$ Tmax divided by paraspinal muscle density, Tmax/vein = Tmax divided by contralateral renal vein density, Tmean = mean density of the tumor

version 19.3.1 (MedCalc Software Ltd.). The normality of distribution of the demographic data and imaging features was assessed using the Kolmogorov-Smirnov and ShapiroWilk tests. Fisher's exact test or Kendall's tau-b test was used to determine the existence of a statistically significant difference between the categorical variables in the CCSK and Wilms' tumor groups. The Mann-Whitney $U$ test was used to compare the continuous variables between the two groups. Categorical data were expressed as counts and percentages, and continuous variables were expressed as the median and range. The intraclass correlation coefficient (ICC) was obtained to evaluate the reliability of the density measurement. Univariable and multivariable logistic regression analyses were performed to determine the diagnostic ability of the significant features for CCSK. The diagnostic performances of the imaging features for identifying CCSK were assessed using receiver operating characteristic (ROC) curve analysis and compared using the area under the curve (AUC). $P$ values less than 0.05 were considered statistically significant for all statistical tests.

\section{RESULTS}

\section{Demographics and Clinical Features}

Thirty patients underwent initial CT imaging outside our hospital. Needle biopsy was used for the pathological confirmation of the tumor in 5 patients from the Wilms' tumor group due to inoperability at the time of diagnosis (bilateral lesions in 2 patients, tumor rupture in 2 patients, and malignant thrombus extension to the inferior vena cava in one patient). Nephrectomy was used for pathological confirmation in all the other patients. Immunohistochemical staining showed that CCSK demonstrated positive INI-1 expression, to exclude rhabdoid tumor and was negative for WT-1, which could exclude Wilms' tumor. The presence of specific curvilinear or arborizing vascular patterns were confirmed using CD34 staining in the CCSK group. The clinical features of both groups are shown in Table 1. The median age at the time of initial CT examination was 2 years (range, $1-8$ years) in the CCSK group and 2 years (range, $0.3-10$ years) in the Wilms' tumor group, which was not significantly different $(p=0.691)$.

The CCSK group consisted of 4 patients with stage 1, 1 patient with stage 2, and 2 patients with stage 4 disease; while the Wilms' tumor group consisted of 28 patients with stage 1, 7 patients with stage 2, 4 patients with stage 3,8 patients with stage 4 disease, and 4 patients with bilateral tumors. Lymph node metastasis was confirmed pathologically in 3 patients (43\%) in the CCSK group and 6 patients $(12 \%)$ in the Wilms' tumor group. The tumor stage 
Table 1. Comparison of Clinical and Imaging Features between the CCSK and Wilms' Tumor Groups

\begin{tabular}{|c|c|c|c|}
\hline & CCSK Group $(n=7)$ & Wilms' Tumor Group $(n=51)$ & $P$ \\
\hline \multicolumn{4}{|l|}{ Clinical features } \\
\hline Age, years & $2(1-8)$ & $2(0.3-10)$ & 0.691 \\
\hline Sex, male:female & $2: 5$ & $30: 21$ & 0.135 \\
\hline Lymph node metastasis & $3(43 \%)$ & $6(12 \%)$ & 0.067 \\
\hline Distant metastasis* & $2(29 \%)$ & $8(16 \%)$ & 0.347 \\
\hline Disease progression during follow-up & $1(14 \%)$ & $8(16 \%)$ & 0.704 \\
\hline \multicolumn{4}{|l|}{ Imaging features } \\
\hline Maximum tumor diameter, cm & $11(8-15)$ & $10(3-24)$ & 0.640 \\
\hline Perinephric engorged vessels & $5(71 \%)$ & $8(16 \%)$ & 0.005 \\
\hline Calcification of tumor & 0 & $4(8 \%)$ & 0.589 \\
\hline Tmean, HU & $98.1(69.5-212.7)$ & $70.9(45.2-192.4)$ & 0.012 \\
\hline Tmax, HU & $148(109-277)$ & $111(69-251)$ & 0.004 \\
\hline Muscle density, HU & $64.9(55.1-82.6)$ & $64.5(43.7-89.6)$ & 0.591 \\
\hline Tmean/muscle & $1.53(1.26-2.66)$ & $1.09(0.77-3.02)$ & 0.002 \\
\hline Tmax/muscle & $2.64(1.70-3.46)$ & $1.67(1.08-3.69)$ & 0.002 \\
\hline Contralateral renal vein density, $\mathrm{HU}$ & $192.7(112.2-264.7)$ & $189.2(93.7-376.1)$ & 0.944 \\
\hline Tmean/vein & $0.53(0.41-0.83)$ & $0.39(0.20-0.71)$ & 0.008 \\
\hline Tmax/vein & $0.94(0.58-1.05)$ & $0.59(0.29-0.87)$ & 0.002 \\
\hline
\end{tabular}

Data are median (range) or number (percentage), if not otherwise specified. $P$ values from Fisher's exact test for categorical variables and Mann-Whitney $U$ test for continuous variables. *Metastasis sites were lung or bone in the CCSK group and lung and/or liver in the Wilms' tumor group. CCSK = clear cell sarcoma of the kidney, HU = Hounsfield unit, Tmax = maximum density of the tumor, Tmax $/$ muscle $=$ Tmax divided by paraspinal muscle density, Tmax/vein = Tmax divided by contralateral renal vein density, Tmean = mean density of the tumor, Tmean $/$ muscle $=$ Tmean divided by paraspinal muscle density, Tmean/vein $=$ Tmean divided by contralateral renal vein density

did not differ significantly between the two groups $(p=$ 0.791). Distant metastases were observed in 2 patients (29\%) with lung or bone metastases in the CCSK group and in 8 patients (16\%) with lung and/or liver metastases in the Wilms' tumor group. The prevalence of pathologic lymph node metastasis and distant metastasis did not differ between the two groups (Table 1 ).

The follow-up interval ranged from 67-2803 days with a median of 1237 days in the CCSK group and 15-3360 days with a median of 994 days in the Wilms' tumor group. Disease progression was observed in 1 patient $(14 \%, 1 / 7)$ in the CCSK group and 8 patients $(16 \%, 8 / 51)$ in the Wilms' tumor group during the follow-up period $(p=0.704)$.

\section{Comparison of Imaging Features between the CCSK and Wilms' Tumor Groups}

The maximum tumor diameter was $8-15 \mathrm{~cm}$ with a median value of $11 \mathrm{~cm}$ in the CCSK group and 3-24 cm with a median value of $10 \mathrm{~cm}$ in the Wilms' tumor group, which was not significantly different $(p=0.640)$.

More than $70 \%$ of patients $(71 \%, 5 / 7)$ in the CCSK group had perinephric vessel engorgement, compared with less than $20 \%(16 \%, 8 / 51)$ patients in the Wilms' tumor group. Engorged perinephric vessels were significantly more frequent in the CCSK group compared to the Wilms' tumor group $(p=0.005)$ (Figs. 1,2$)$. The age $(p=0.354)$ or tumor stage $(p=0.608)$ of patients with and without perinephric engorged vessels did not differ significantly. However, tumor size was larger in patients with engorged perinephric vessels (median, $12 \mathrm{~cm}[9-24 \mathrm{~cm}]$ vs. $10 \mathrm{~cm}[3-15 \mathrm{~cm}$, $p=0.038$ ) only in the Wilms' tumor group.

Intratumoral calcification was not observed in the CCSK group but was observed in 4 patients in the Wilms' tumor group. Almost all tumors in both groups, except one case of Wilms' tumor, showed a heterogeneous enhancement pattern. Venous tumor thrombus extension was observed in only 2 patients in the Wilms' tumor group, of which 1 patient also had engorged perinephric vessels. Tumor rupture was only seen in 5 cases in the Wilms' tumor group.

The ICCs for reliability of the density measurement were 0.963 (95\% confidence interval [CI], 0.937-0.978) for Tmean, 0.949 (95\% CI, 0.914-0.970) for Tmax, 0.840 (95\% CI, 0.729-0.905) for paraspinal muscle density, and $0.943(95 \%$ CI, 0.903-0.966) for contralateral renal vein density (all, $p<0.001$ ). Only the first measurement data were used in the density study, based on these good-toexcellent reliability results. Among the measured density values, both Tmean and Tmax were significantly higher 
in the CCSK group than those in the Wilms' tumor group (median, 98.1 vs. 70.9 Hounsfield unit [HU] for Tmean and 148.0 vs. $111.0 \mathrm{HU}$ for Tmax; $p=0.012$ and 0.004 for each) (Table 1). However, the paraspinal muscle density and contralateral renal vein density did not differ significantly. Both the Tmean/muscle (median, 1.53 vs. $1.09 ; p=0.002$ ) and Tmax/muscle (median, 2.64 vs. $1.67 ; p=0.002$ ) ratios were also significantly higher in the CCSK group than those in the Wilms' tumor group (Fig. 3). The Tmean/vein (median,

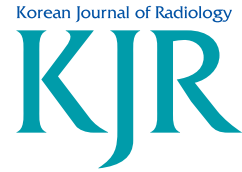

0.53 vs. $0.39 ; p=0.008$ ) and Tmax/vein (median, 0.94 vs. $0.59 ; p=0.002$ ) (Table 1) ratios were also higher in the CCSK group than those in the Wilms' tumor group.

\section{Assessment of Diagnostic Performances for CCSK Diagnosis}

Univariable logistic regression analyses were performed using the imaging features that differed significantly between the two groups, i.e., perinephric vessel
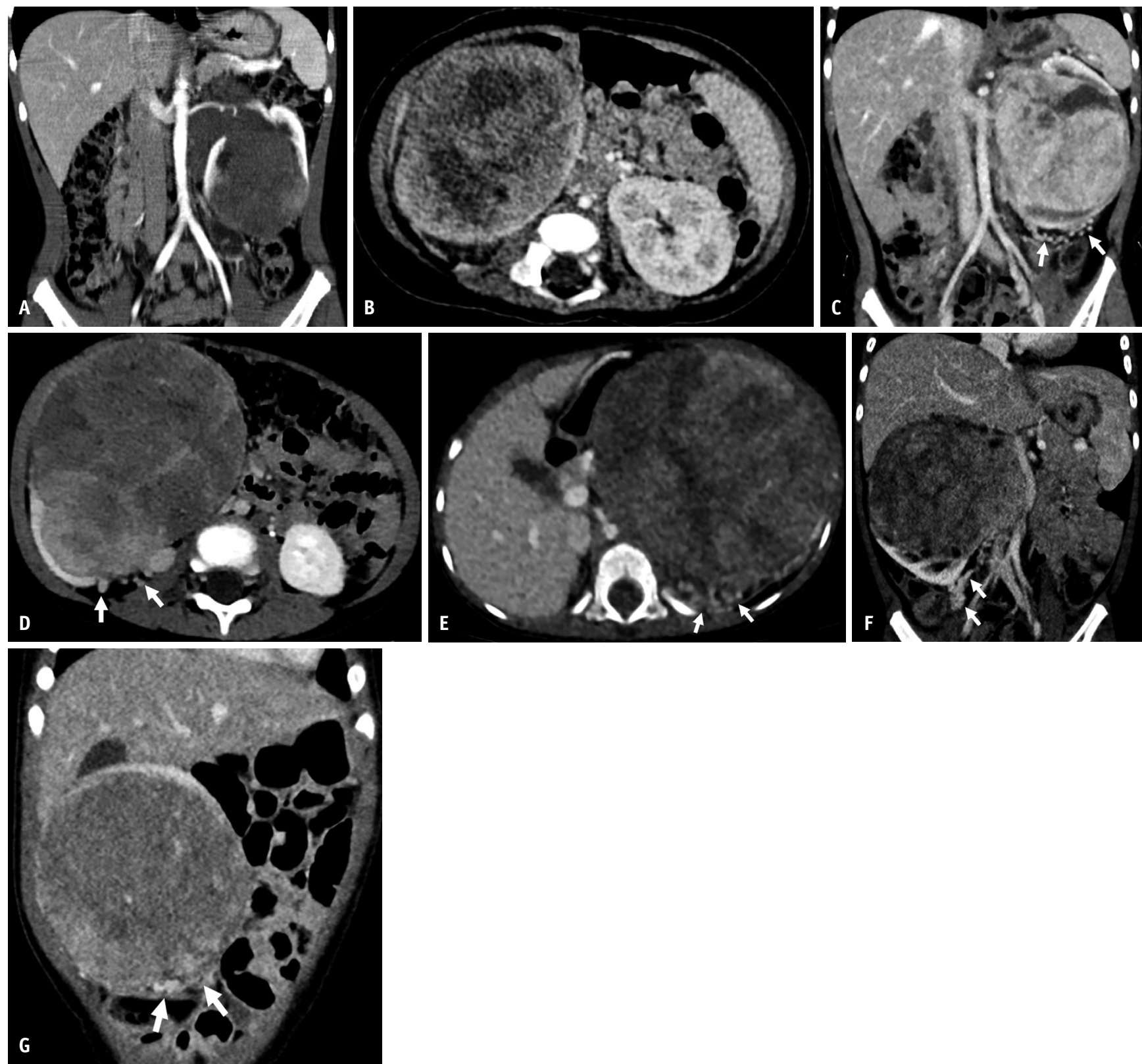

Fig. 2. Patients with clear cell sarcoma of the kidney.

A-G. The representative CT images demonstrate heterogeneously enhancing renal masses without $(\mathbf{A}, \mathbf{B})$ and with (C-G) engorged perinephric vessels (arrows) in patients with clear cell sarcoma of the kidney. (A) An 8-year-old boy, (B) a 1-year-old boy, (C) a 3-year-old girl (same patient as Fig. 1), (D) a 2-year-old girl, (E) a 2-year-old girl, (F) a 2-year-old girl, and (G) a 1-year-old girl. 
engorgement and density variables. Perinephric vessel engorgement, Tmean, Tmax, Tmean/muscle, Tmax/muscle, Tmean/vein, and Tmax/vein were significant parameters (Table 2). Multivariable analyses also demonstrated the significance of perinephric vessel engorgement (odds ratio, 13.615 [95\% CI, 1.770-104.730], $p=0.012$ ) and Tmax/ muscle (odds ratio 5.881 [95\% CI, 1.337-25.871], $p=$ 0.019). Tmean, Tmax, Tmean/muscle, Tmean/vein, and Tmax/vein were not included in the multivariable analysis due to the possible confounding effect of Tmax/muscle.

The AUC values (95\% CI) used to evaluate the diagnostic performance of these features were $0.822(0.699-0.910)$
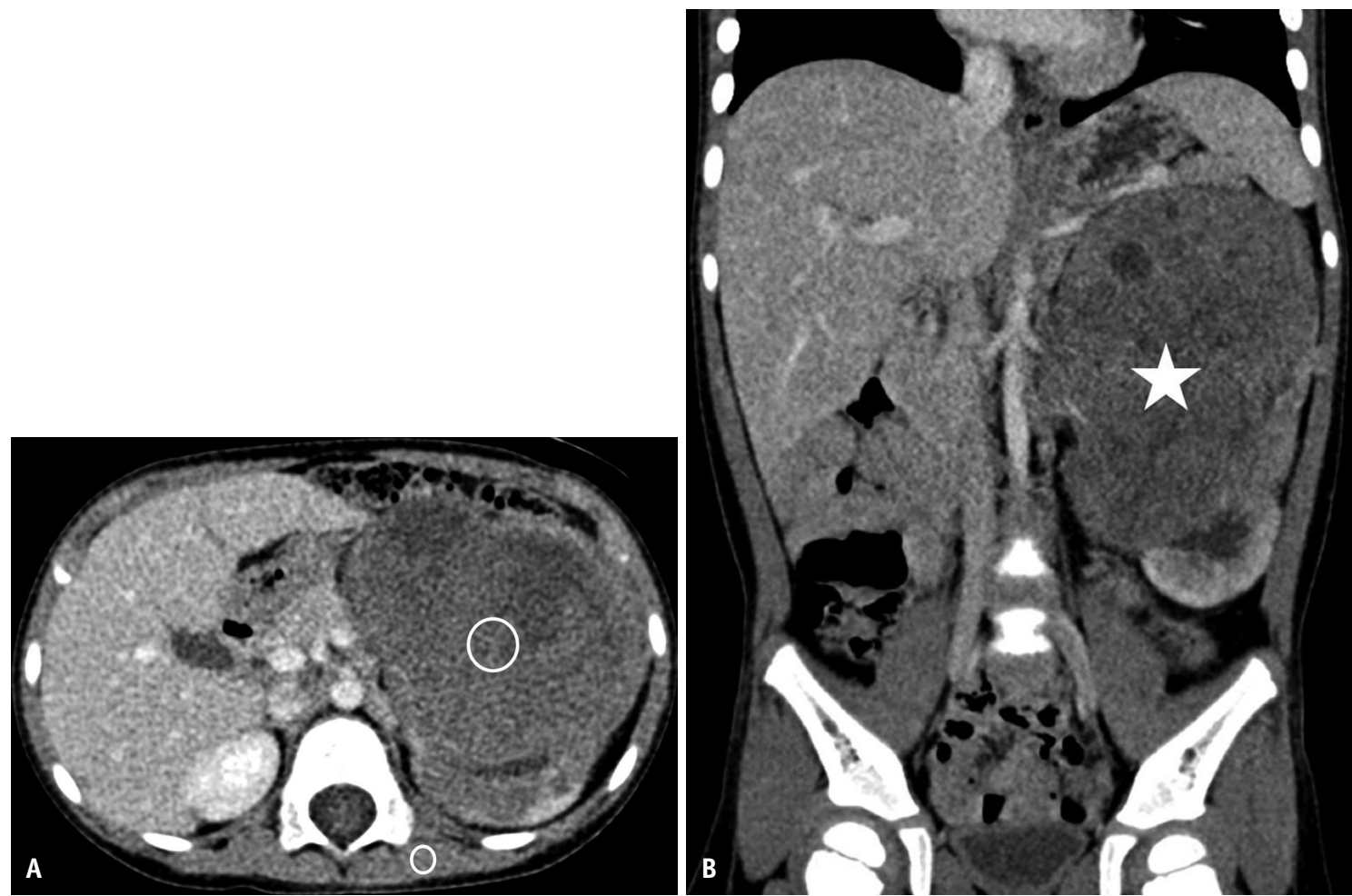

Fig. 3. A 3-year-old boy with a left renal mass confirmed as Wilms' tumor.

A, B. The axial (A) and coronal (B) contrast-enhanced abdominal-pelvic CT images in the renal parenchymal phase demonstrate a large, heterogeneous mass (star) replacing the left kidney tissue without perinephric vessel engorgement, showing less enhancement than the renal parenchyma. Regions of interest (circles) were marked on the axial image to obtain the mean (Tmean) and maximum (Tmax) tumor densities of the most enhancing solid portion and mean density of the paraspinal muscle. The Tmean was $47.5 \mathrm{HU}$, Tmax was $95.0 \mathrm{HU}$, paraspinal muscle density was $61.2 \mathrm{HU}$, and contralateral renal vein density was $145.6 \mathrm{HU}$. Both Tmax/muscle $(1.55<1.97)$ and Tmax/vein $(0.65<0.76)$ were lower than the suggested cutoff values. $\mathrm{HU}=$ Hounsfield unit, Tmax = maximum density of the tumor, Tmax/muscle = Tmax divided by paraspinal muscle density, Tmax/vein = Tmax divided by contralateral renal vein density, Tmean = mean density of the tumor

Table 2. Univariable and Multivariable Logistic Regression Analyses for the Diagnosis of CCSK

\begin{tabular}{|c|c|c|c|c|c|c|c|c|c|}
\hline \multirow{2}{*}{ Variables } & \multicolumn{3}{|c|}{ Univariable Analysis } & \multicolumn{6}{|c|}{ Multivariable Analysis } \\
\hline & OR & $95 \% \mathrm{CI}$ & $P$ & OR & $95 \%$ CI & $P$ & OR & $95 \% \mathrm{CI}$ & $P$ \\
\hline Engorged vessels & 13.438 & $2.210-81.714$ & 0.005 & 13.615 & $1.770-104.730$ & 0.012 & 4.018 & $0.456-35.412$ & 0.019 \\
\hline Tmean, HU & 1.022 & $1.004-1.040$ & 0.019 & & & & & & \\
\hline Tmax, HU & 1.023 & $1.006-1.040$ & 0.009 & & & & & & \\
\hline Tmean/muscle & 4.557 & $1.181-17.585$ & 0.028 & & & & & & \\
\hline Tmax/muscle & 5.723 & $1.540-21.263$ & 0.009 & 5.881 & $1.337-25.871$ & 0.019 & & & \\
\hline Tmean/vein, $x 10^{*}$ & 2.603 & $1.389-4.877$ & 0.003 & & & & & & \\
\hline Tmax/vein, x 10* & 3.116 & $1.488-6.523$ & 0.003 & & & & 2.588 & $1.171-5.723$ & 0.002 \\
\hline
\end{tabular}

*10 multiplied values were evaluation for these variables. CCSK = clear cell sarcoma of the kidney, $\mathrm{CI}=$ confidence interval, $\mathrm{HU}=$ Hounsfield unit, $\mathrm{OR}=$ odds ratio, Tmax = maximum density of the tumor, Tmax/muscle = Tmax divided by paraspinal muscle density, Tmax/vein = Tmax divided by contralateral renal vein density, Tmean = mean density of the tumor, Tmean $/$ muscle $=$ Tmean divided by paraspinal muscle density, Tmean/vein = Tmean divided by contralateral renal vein density 
for Tmax and 0.845 (0.725-0.926) for Tmax/muscle, which were not significantly different $(p=0.657)$. A cutoff value of 1.97 for Tmax/muscle showed $86 \%$ sensitivity and $77 \%$ specificity for the diagnosis of CCSK $(p<0.001)$ (Fig. 4).

The cutoff value of Tmax was $134 \mathrm{HU}$ with $71 \%$ sensitivity and $84 \%$ specificity. The results of the other density parameters are presented in Table 3. The AUC values of these parameters were not different.

The AUC value ( $95 \%$ CI) for the combination of Tmax/ muscle and perinephric vessel engorgement was 0.887

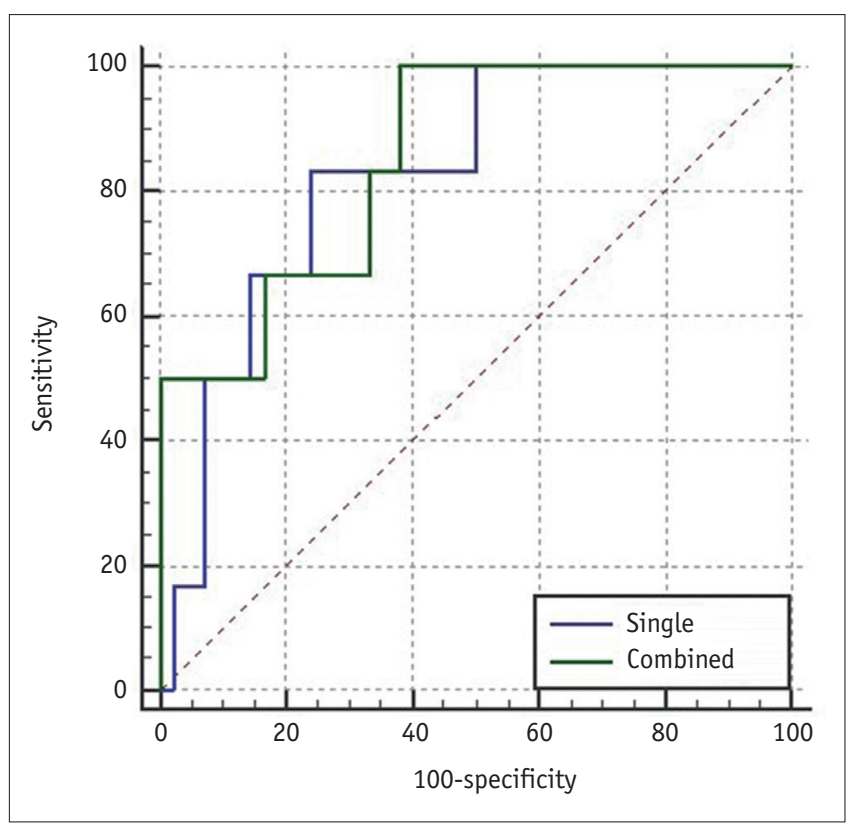

Fig. 4. Receiver operating characteristic curves for the diagnosis of clear cell sarcoma of the kidney. Two analyses were performed using a single variable, i.e., Tmax/muscle and combined variables, i.e., Tmax/muscle and perinephric vessel engorgement. The values of the area under the curve ( $95 \%$ confidence interval) were $0.845(0.725-0.926 ; p<0.001)$ for the single variable and $0.887(0.776-0.955 ; p<0.001)$ for the combined variables, without statistical difference $(p=0.524)$. Tmax $/$ muscle $=$ Tmax divided by paraspinal muscle density
(0.776-0.955) $(p<0.001)$, which was slightly higher than that for Tmax/muscle, without any significant difference in the pairwise comparisons of the two ROC curves $(p=0.524)$.

\section{DISCUSSION}

CCSK is an aggressive malignant renal tumor that requires chemotherapy with doxorubicin, which is associated with the risks of cardiotoxicity, nephrotoxicity, and reduced fertility. Therefore, the differential diagnosis of CCSK from Wilms' tumor is an essential task. In this study, various clinical and CT imaging features of CCSK were compared with those of Wilms' tumor using a retrospective review. No significant differences were observed between the clinical features of the two groups. However, imaging features such as perinephric vessel engorgement and higher tumor density values were features that distinguished CCSK from Wilms' tumor. A Tmax/muscle cutoff value of 1.97 showed $86 \%$ sensitivity and $77 \%$ specificity for the diagnosis of CCSK. Therefore, we determined that these findings were helpful in differentiating CCSK from Wilms' tumors.

Wilms' tumor usually appears as a large, heterogeneous intrarenal mass on abdominal CT. These tumors demonstrate a lower degree of enhancement compared to the adjacent normal renal parenchyma [5]. Necrosis and hemorrhage are commonly observed in the intratumoral portion, and calcifications are seen in approximately $15 \%$ of Wilms' tumors [11,12]. However, these features may also be observed in CCSK [8]. CCSK also usually appears as a heterogeneously enhancing solid renal mass with cysts on abdominal CT. Currently, bone metastasis is the only known indicator of CCSK that distinguishes it from Wilms' tumor [9]. None of the known imaging features can distinguish between CCSK and Wilms' tumor in patients without bone metastasis $[5,13]$.

Table 3. Diagnostic Performance of Density Parameters for the Diagnosis of CCSK

\begin{tabular}{lcccccc}
\hline \multicolumn{1}{c}{ Variables } & AUC & 95\% Confidence Interval & $P$ & Cutoff Value & Sensitivity (\%) & Specificity (\%) \\
\hline Tmean, HU & 0.790 & $0.663-0.886$ & $<0.001$ & $>84$ & 85.7 & 74.5 \\
Tmax, HU & 0.822 & $0.699-0.910$ & $<0.001$ & $>134$ & 71.4 & 84.3 \\
Tmean/muscle & 0.840 & $0.721-0.923$ & $<0.001$ & $>1.25$ & 100 & 64.7 \\
Tmax/muscle & 0.845 & $0.725-0.926$ & $<0.001$ & $>1.97$ & 85.7 & 76.5 \\
Tmean/vein & 0.804 & $0.679-0.897$ & $<0.001$ & $>0.4$ & 71.4 & 84.9 \\
Tmax/vein & 0.847 & $0.729-0.928$ & $<0.001$ & $>0.76$ & 76.3 \\
\hline
\end{tabular}

$\mathrm{AUC}=$ area under the curve, CCSK = clear cell sarcoma of the kidney, HU = Hounsfield unit, Tmax = maximum density of the tumor, Tmax/ muscle $=$ Tmax divided by paraspinal muscle density, Tmax/vein = Tmax divided by contralateral renal vein density, Tmean = mean density of the tumor, Tmean/muscle = Tmean divided by paraspinal muscle density, Tmean/vein = Tmean divided by contralateral renal vein density 
CCSK often presents as a large, well-circumscribed, sharply demarcated mass on gross pathological examination [7]. These tumors arise from the medullary region of the kidney and replace normal renal tissue [14]. The microscopic features include nests or cords of small, plump cells separated by networks of delicate fibrovascular septa. The blood vessels in the fibrovascular septa contain regular branching capillaries, which exhibit a chickenwire pattern, which is typical of CCSK $[1,15]$. Although CCSK is a histologically diverse tumor with myxoid $(50 \%)$, sclerosing $(35 \%)$, cellular $(26 \%)$, epithelioid $(13 \%)$, spindle $(7 \%)$, storiform $(4 \%)$, and anaplastic $(2.6 \%)$ patterns [1], the distinctive complex vascular network can be a helpful diagnostic finding. Our study hypothesized that the distinctive vasculature of CCSK may manifest as distinct imaging findings on $\mathrm{CT}$, which could differentiate it from Wilms' tumor. We found a higher frequency of peritumoral vessel engorgement and higher degree of tumor enhancement in CCSK.

Other hypervascular renal tumors can present with hyperenhancement on CT and peritumoral neovascularity. One study reported that sarcomatoid renal cell carcinomas demonstrated larger tumor size and larger peritumoral vessels compared to clear cell carcinomas in adults [16]. Although rare in childhood, renal cell carcinoma may occur in patients aged 15 to 19 years. Renal clear cell carcinoma [17], alveolar soft part sarcoma arising from the kidney [18], and solitary fibrous tumor from the retroperitoneum [19] can also present with prominent feeding vessels. However, no reports were found on peritumoral vascular engorgement associated with CCSK in children. To the best of our knowledge, this is the first study to identify more frequent peritumoral vascular engorgement and greater internal enhancement of the solid portion of the CCSK compared to Wilms' tumor in children. Although we could not directly compare the imaging findings with the pathologic results, our tumor density evaluation demonstrated good-toexcellent reliability and showed consistent results when normalized with paraspinal muscle density and contralateral renal vein density. Perinephric vessel engorgement was not associated with the patient's age, tumor size, or disease stage in the CCSK group, even though larger tumor size was associated with perinephric vessel engorgement in the Wilms' tumor group. Additional studies are needed to correlate imaging and pathological findings.

Our study had several limitations. First, the study population was small, owing to the rarity of CCSK in children. Thus, the study included only 7 patients with CCSK, despite the 10-year study period. Second, our study included CT scans that were obtained outside our hospital using different protocols. All the included CT scans were in renal parenchymal phase, but the timing or amount of contrast agents used could have been different. We used the enhancement ratio to correct for the variations in the enhancement timing, which demonstrated significant differences between CCSK and Wilms' tumors. However, further studies with optimal enhancement timing are needed. Third, we could not directly compare the imaging findings and results of the pathological examination in this retrospective study. We hypothesized that the imaging findings could be different because of the greater hypervascular character of CCSK compared to Wilms' tumor. However, CCSK has several subtypes, and Wilms' tumors can also be hypervascular, as evidenced by the peritumoral vessel engorgement observed in some patients in this study. Further prospective studies with direct comparison of the imaging and pathological findings are needed.

In conclusion, imaging features such as perinephric vessel engorgement and higher tumor enhancement (Tmax/muscle $>1.97$ ) were helpful in differentiating CCSK from Wilms, tumor in children with primary renal tumors under 10 years of age.

\section{Conflicts of Interest}

The authors have no potential conflicts of interest to disclose.

\section{Author Contributions}

Conceptualization: Mi-Jung Lee. Data curation: Choeum Kang, Hyun Joo Shin, Haesung Yoon, Mi-Jung Lee. Formal analysis: Choeum Kang, Hyun Joo Shin, Haesung Yoon, MiJung Lee. Investigation: Jung Woo Han, Chuhl Joo Lyu, Mi-Jung Lee. Methodology: Choeum Kang, Mi-Jung Lee. Project administration: Mi-Jung Lee. Resources: Jung Woo Han, Chuhl Joo Lyu, Mi-Jung Lee. Software: Mi-Jung Lee. Supervision: Mi-Jung Lee. Validation: Choeum Kang, Mi-Jung Lee. Visualization: Choeum Kang, Mi-Jung Lee. Writingoriginal draft: Choeum Kang, Hyun Joo Shin, Haesung Yoon, Mi-Jung Lee. Writing - review \& editing: Choeum Kang, Hyun Joo Shin, Haesung Yoon, Mi-Jung Lee.

\section{ORCID iDs}

Choeum Kang

https://orcid.org/0000-0002-0958-188X 
Hyun Joo Shin

https://orcid.org/0000-0002-7462-2609

Haesung Yoon

https://orcid.org/0000-0003-0581-8656

Jung Woo Han

https://orcid.org/0000-0001-8936-1205

Chuhl Joo Lyu

https://orcid.org/0000-0001-7124-7818

Mi-Jung Lee

https://orcid.org/0000-0003-3244-9171

\section{REFERENCES}

1. Argani P, Perlman EJ, Breslow NE, Browning NG, Green DM, D'Angio GJ, et al. Clear cell sarcoma of the kidney: a review of 351 cases from the National Wilms Tumor Study Group Pathology Center. Am J Surg Pathol 2000;24:4-18

2. Furtwängler R, Gooskens SL, van Tinteren H, de Kraker J, Schleiermacher G, Bergeron C, et al. Clear cell sarcomas of the kidney registered on International Society of Pediatric Oncology (SIOP) 93-01 and SIOP 2001 protocols: a report of the SIOP Renal Tumour Study Group. Eur J Cancer 2013;49:3497-3506

3. Franco A, Dao TV, Lewis KN, Biddinger PW. A case of clear cell sarcoma of the kidney. J Radiol Case Rep 2011;5:8-12

4. Seibel NL, Li S, Breslow NE, Beckwith JB, Green DM, Haase GM, et al. Effect of duration of treatment on treatment outcome for patients with clear-cell sarcoma of the kidney: a report from the National Wilms' Tumor Study Group. J Clin Oncol 2004;22:468-473

5. Chung EM, Graeber AR, Conran RM. Renal tumors of childhood: radiologic-pathologic correlation part 1 . The 1st decade: from the radiologic pathology archives. Radiographics 2016;36:499522

6. Sebire NJ, Vujanic GM. Paediatric renal tumours: recent developments, new entities and pathological features. Histopathology 2009;54:516-528

7. Gooskens SL, Furtwängler R, Vujanic GM, Dome JS, Graf N, van den Heuvel-Eibrink MM. Clear cell sarcoma of the kidney: a review. Eur J Cancer 2012;48:2219-2226

8. Glass RB, Davidson AJ, Fernbach SK. Clear cell sarcoma of the kidney: CT, sonographic, and pathologic correlation. Radiology 1991;180:715-717

9. Sleight G, Lock MM. Clear cell sarcoma of the kidney: a renal tumor of childhood that metastasizes to bone. AJR Am J Roentgenol 1986;146:64-66

10. Aw SJ, Chang KTE. Clear cell sarcoma of the kidney. Arch Pathol Lab Med 2019;143:1022-1026

11. Lee EY. CT imaging of mass-like renal lesions in children. Pediatr Radiol 2007;37:896-907

12. Brisse HJ, Smets AM, Kaste SC, Owens CM. Imaging in unilateral Wilms tumour. Pediatr Radiol 2008;38:18-29

13. Stanescu AL, Acharya PT, Lee EY, Phillips GS. Pediatric renal neoplasms: MR imaging-based practical diagnostic approach. Magn Reson Imaging Clin N Am 2019;27:279-290

14. Balarezo FS, Joshi VV. Clear cell sarcoma of the pediatric kidney: detailed description and analysis of variant histologic patterns of a tumor with many faces. Adv Anat Pathol 2001;8:98-108

15. Boo YJ, Fisher JC, Haley MJ, Cowles RA, Kandel JJ, Yamashiro DJ. Vascular characterization of clear cell sarcoma of the kidney in a child: a case report and review. J Pediatr Surg 2009;44:2031-2036

16. Schieda N, Thornhill RE, Al-Subhi M, McInnes MD, Shabana WM, van der Pol CB, et al. Diagnosis of sarcomatoid renal cell carcinoma with $\mathrm{CT}$ : evaluation by qualitative imaging features and texture analysis. AJR Am J Roentgenol 2015;204:10131023

17. Zhang J, Lefkowitz RA, Wang L, Ishill NM, Moskowitz CS, Russo $\mathrm{P}$, et al. Significance of peritumoral vascularity on CT in evaluation of renal cortical tumor. J Comput Assist Tomogr 2007;31:717-723

18. Kim JM, Im SA, Oh SN, Chung NG. Alveolar soft part sarcoma arising from the kidney: imaging and clinical features. Korean J Radiol 2014;15:381-385

19. Messiou C, Moskovic E, Vanel D, Morosi C, Benchimol R, Strauss $D$, et al. Primary retroperitoneal soft tissue sarcoma: imaging appearances, pitfalls and diagnostic algorithm. Eur J Surg Oncol 2017;43:1191-1198 